Toxicomics Report

\title{
Metabolomic analysis of low molecular weight substances released into medium from HEK293 cells treated with methylmercury
}

\author{
Takashi Toyama, Gi-Wook Hwang and Akira Naganuma \\ Laboratory of Molecular and Biochemical Toxicology, Graduate School of Pharmaceutical Sciences, \\ Tohoku University, Sendai 980-8575, Japan
}

(Received November 28, 2015; Accepted December 3, 2015)

\begin{abstract}
This study attempted to identify substances that are driven out of HEK293 cells by methylmercury. Metabolomic analysis revealed that the levels of 3-phenylpropionic acid, citrulline, lactic acid, ornithine, proline and beta-alanine in the cell culture medium were increased by the treatment of cells with methylmercury. Address to the mechanism underlying the release of these substances will provide useful information to elucidate the toxicity mechanism of methylmercury.
\end{abstract}

Key words: Methylmercury, Metabolomic analysis, Substances released from cells, HEK293 cells

\section{INTRODUCTION}

Methylmercury is a well-known environmental pollutant that induces disorders in central nervous system (Bakir et al., 1973; Harada, 1995). It is presumable that methylmercury affect release of endogenous substances (e.g. amino acids, saccharides and nucleotides) from the cells (Aschner et al., 1994), while there is little evidence. In this study, we performed metabolomic analysis to identify low molecular weight substances that are released into medium from HEK293 cells treated with methylmercury.

\section{MATERIALS AND METHODS}

\section{Materials}

Methylmercuric chloride was purchased from Sigma-Aldrich (St. Louis, MO, USA). InartSep C18 was obtained from GL Science (Tokyo, Japan). All other reagents used were of the highest grade available.

\section{Cells and cell culture}

Human embryonic kidney HEK293 cells were maintained in Dulbecco's modified Eagle's medium (10\% fetal bovine serum, $0.3 \%$ L-glutamine and antibiotics (100 U/mL penicillin and $100 \mu \mathrm{g} / \mathrm{mL}$ streptomycin)). HEK293 cells were grown at $37^{\circ} \mathrm{C}$ in a humidified incubator under an atmosphere of $\mathrm{CO}_{2}(5 \%)$ and room air (95\%).

\section{Metabolomic analysis}

HEK 293 cells were seeded on a $10 \mathrm{~cm}$ dish at a cell concentration $6 \times 10^{6} \mathrm{cells} / \mathrm{dish}$, and exposed to methylmercuric chloride $(50 \mu \mathrm{M})$ for $2 \mathrm{hr}$. The cells were washed twice with PBS, transferred to fresh medium and incubated for additional $6 \mathrm{hr}$. The medium was collected and filtrated by syringe filter $(0.2 \mu \mathrm{m}$ pore size) (Corning, NY, USA). An aliquot of the medium $(10 \mathrm{~mL})$ was loaded onto a C18 (ODS) solid phase extraction column (GL Science, Tokyo, Japan), which was equilibrated by DDW (Toyama et al., 2015). ODS flow through fraction was collected and subjected to metabolomic analysis. Metabolomics was performed using CE-TOF/MS in both positive and negative modes as we previously reported (Hwang et al., 2013). Briefly, CE-TOF/MS was performed using the Agilent CE Capillary Electrophoresis System and data analysis was performed using software from Human Metabolome Technologies Inc. (Yamagata, Japan). We detected 51 compounds (positive ion 36 and negative ion 15) from the Basic Scan library. The compounds increased more than 1.3 fold compare to control were listed in the table.

\section{Statistical analysis}

Statistical significance was assessed by the $t$-test. All $p$ values are two tailed.

Correspondence: Akira Naganuma (E-mail: naganuma@m.tohoku.ac.jp) 
T. Toyama et al.

\section{RESULTS AND DISCUSSION}

To identify the substances that are driven out of the cells by methylmercury, HEK293 cells were cultured in medium containing methylmercury for $2 \mathrm{hr}$. After washing with PBS, the cells were further cultured for $6 \mathrm{hr}$ in fresh methylmercury-free medium. The medium was collected, passed through ODS column to eliminate ODSbinding compounds (Toyama et al., 2015) and subjected to metabolomic analysis. Metabolomic analysis of low molecular weight compounds in the fraction showed that the concentrations of 3-phenylpropionic acid, citrulline, lactic acid, ornithine, proline and beta-alanine increased 1.3 times or more if the cells were treated with methylmercury (Table 1). The fact that these six substances are driven out of the cell by methylmercury is interesting in terms of assessing the influence of methylmercury on cellular functions. Elucidation of the mechanism underlying the release of these substances will contribute to understanding of the effect of methylmercury on cellular functions.

\section{ACKNOWLEDGMENTS}

This work was supported by a Grant-in-Aid (15H05714) for scientific research from the Ministry of Education, Culture, Sports, Science and Technology of Japan.

Conflict of interest---- The authors declare that there is no conflict of interest.
Table 1. Compounds whose release into medium was increased by treatment of HEK293 cells with methylmercury.

\begin{tabular}{lc}
\hline Compound & Ratio (Control vs methylmercury) \\
\hline Proline & 1.85 \\
Ornithine & 1.59 \\
3-Alanine & 1.45 \\
Lactic acid & 1.43 \\
Citrulline & 1.32 \\
3-Phenylpropionic acid & 1.30 \\
\hline
\end{tabular}

\section{REFERENCES}

Aschner, M., Mullaney, K.J., Wagoner, D., Lash, L.H. and Kimelberg, H.K. (1994): Intracellular glutathione (GSH) levels modulate mercuric chloride (MC)- and methylmercuric chloride $(\mathrm{MeHgCl})$-induced amino acid release from neonatal rat primary astrocytes cultures. Brain Res., 664, 133-140.

Bakir, F., Damluji, S.F., Amin-Zaki, L., Murtadha, M., Khalidi, A., Al-Rawi, N.Y., Tikriti, S., Dahahir, H.I., Clarkson, T.W., Smith, J.C. and Doherty, R.A. (1973): Methylmercury poisoning in Iraq. Science, 181, 230-241.

Harada, M. (1995): Minamata disease: methylmercury poisoning in Japan caused by environmental pollution. Crit. Rev. Toxicol., 25, $1-24$.

Hwang, G.W., Lee, J.Y., Kim, M.S., Sato, M., Takahashi, T. and Naganuma, A. (2013): Changes in the levels of low molecular weight metabolites in the mouse cerebellum following treatment with methylmercury. J. Toxicol. Sci., 38, 703-706.

Toyama, T., Murakami, S., Kuge, S., Hwang, G.W. and Naganuma, A. (2015): Methylmercury induces release of a cytotoxic factor from HEK293 cells into medium. Fundam. Toxicol. Sci., 2, 223226. 